\title{
MULTIWAVELENGTH EVIDENCE FOR QUASI-PERIODIC MODULATION IN THE GAMMA-RAY BLAZAR PG 1553+113
}

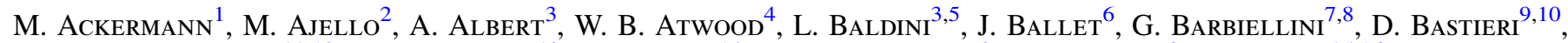
J. Becerra Gonzalez ${ }^{11,12}$, R. Bellazzini ${ }^{13}$, E. Bissaldi ${ }^{14}$, R. D. Blandford ${ }^{3}$, E. D. Bloom ${ }^{3}$, R. Bonino ${ }^{15,16}$, E. BotTaCini ${ }^{3}$, J. Bregeon ${ }^{17}$, P. Bruel ${ }^{18}$, R. Buehler ${ }^{1}$, S. Buson ${ }^{9,10}$, G. A. Caliandro ${ }^{3,19}$, R. A. Cameron ${ }^{3}$, R. CAPuto ${ }^{4}$, M. Caragiulo ${ }^{14}$, P. A. Caraveo ${ }^{20}$, E. Cavazzuti ${ }^{21}$, C. Cecchi ${ }^{2,23}$, A. CheKhtman ${ }^{24}$, J. Chiang ${ }^{3}$, G. Chiaro ${ }^{10}$, S. Ciprini ${ }^{21,22,25}$, J. Cohen-Tanugi ${ }^{17}$, J. Conrad ${ }^{26,27,28}$, S. Cutini ${ }^{21,22,25}$, F. D’Ammando ${ }^{29,30}$, A. DE Angelis ${ }^{31}$, F. De Palma ${ }^{14,32}$ ', R. Desiante ${ }^{15,33}$, L. Di Venere ${ }^{34}$, A. Domínguez ${ }^{2}$, P. S. Drell ${ }^{3}$, C. Favuzzi ${ }^{14,34}$, S. J. Fegan ${ }^{18}$, E. C. Ferrara ${ }^{11}$, W. B. Focke ${ }^{3}$, L. Fuhrmann $^{35}$, Y. Fukazawa ${ }^{36}$, P. Fusco ${ }^{14,34}$, F. Gargano ${ }^{14}$, D. Gasparrini ${ }^{21,22,25}$, N. Giglietto ${ }^{14,34}$, P. Giommi ${ }^{21}$, F. Giordano $^{14,34}$, M. Giroletti ${ }^{29}$, G. Godfrey ${ }^{3}$, D. Green ${ }^{11,12}$, I. A. Grenier ${ }^{6}$, J. E. Grove ${ }^{37}$, S. Guiriec ${ }^{11,64}$,

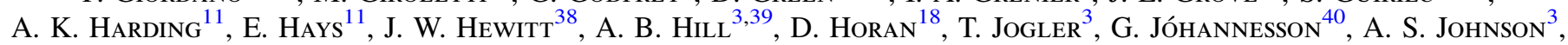

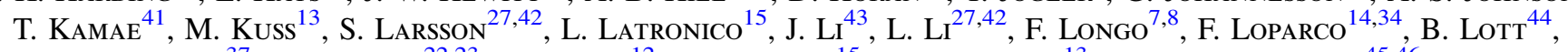
M. N. Lovellette ${ }^{37}$, P. Lubrano ${ }^{22,23}$, J. Magill ${ }^{12}$, S. Maldera ${ }^{15}$, A. Manfreda ${ }^{13}$, W. Max-Moerbeck ${ }^{45,46}$, M. Mayer $^{1}$, M. N. Mazziotta ${ }^{14}$, J. E. McEnery ${ }^{11,12}$, P. F. Michelson ${ }^{3}$, T. Mizuno ${ }^{47}$, M. E. Monzani ${ }^{3}$, A. Morselli ${ }^{48}$, I. V. Moskalenkoo ${ }^{3}$, S. Murgia ${ }^{49}$, E. Nuss ${ }^{17}$, M. Ohno ${ }^{36}$, T. Ohsugi ${ }^{47}$, R. Ojha ${ }^{11}$, N. OmodeI ${ }^{3}$, E. Orlando ${ }^{3}$, J. F. Ormes ${ }^{50}$, D. Paneque ${ }^{3,51}$, T. J. Pearson ${ }^{46}$, J. S. Perkins ${ }^{11}$, M. Perri ${ }^{21,25}$, M. Pesce-Rollins ${ }^{3,13}$, V. Petrosian ${ }^{3}$, F. Piron ${ }^{17}$, G. Pivato ${ }^{13}$, T. A. Porter ${ }^{3}$, S. Rainò ${ }^{14,34}$, R. Rando ${ }^{9,10}$, M. Razzano ${ }^{13,65}$, A. Readhead ${ }^{46}$, A. Reimer ${ }^{3,52}$, O. Reimer ${ }^{3,52}$, A. Schulz ${ }^{1}$, C. Sgrò ${ }^{13}$,

E. J. Siskind ${ }^{53}$, F. Spada ${ }^{13}$, G. Spandre ${ }^{13}$, P. Spinelli ${ }^{14,34}$, D. J. Suson ${ }^{54}$, H. Takahashi ${ }^{36}$, J. B. Thayer ${ }^{3}$, D. J. Thompson ${ }^{11}$, L. Tibaldo ${ }^{3}$, D. F. Torres ${ }^{43,55}$, G. Tosti ${ }^{22,23}$, E. Troja ${ }^{11,12}$, Y. UchiYama ${ }^{56}$, G. Vianello ${ }^{3}$, K. S. Wood ${ }^{37}$, M. Wood $^{3}$,

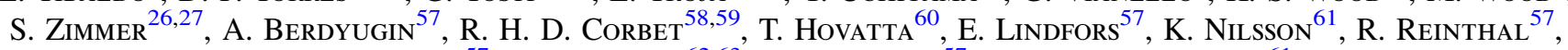

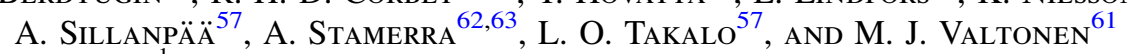

${ }^{1}$ Deutsches Elektronen Synchrotron DESY, D-15738 Zeuthen, Germany

2 Department of Physics and Astronomy, Clemson University, Kinard Lab of Physics, Clemson, SC 29634-0978, USA

${ }^{3}$ W. W. Hansen Experimental Physics Laboratory, Kavli Institute for Particle Astrophysics and Cosmology, Department of Physics and SLAC National Accelerator Laboratory, Stanford University, Stanford, CA 94305, USA

${ }^{4}$ Santa Cruz Institute for Particle Physics, Department of Physics and Department of Astronomy and Astrophysics, University of California at Santa Cruz, Santa Cruz, CA 95064, USA

${ }^{5}$ Università di Pisa and Istituto Nazionale di Fisica Nucleare, Sezione di Pisa I-56127 Pisa, Italy

${ }^{6}$ Laboratoire AIM, CEA-IRFU/CNRS/Université Paris Diderot, Service d'Astrophysique, CEA Saclay, F-91191 Gif sur Yvette, France

${ }^{7}$ Istituto Nazionale di Fisica Nucleare, Sezione di Trieste, I-34127 Trieste, Italy

${ }^{8}$ Dipartimento di Fisica, Università di Trieste, I-34127 Trieste, Italy

${ }^{9}$ Istituto Nazionale di Fisica Nucleare, Sezione di Padova, I-35131 Padova, Italy

${ }^{10}$ Dipartimento di Fisica e Astronomia "G. Galilei," Università di Padova, I-35131 Padova, Italy

${ }^{11}$ NASA Goddard Space Flight Center, Greenbelt, MD 20771, USA; David.J.Thompson@nasa.gov

12 Department of Physics and Department of Astronomy, University of Maryland, College Park, MD 20742, USA

${ }_{13}$ Istituto Nazionale di Fisica Nucleare, Sezione di Pisa, I-56127 Pisa, Italy

${ }^{14}$ Istituto Nazionale di Fisica Nucleare, Sezione di Bari, I-70126 Bari, Italy

${ }^{15}$ Istituto Nazionale di Fisica Nucleare, Sezione di Torino, I-10125 Torino, Italy

${ }_{17}^{16}$ Dipartimento di Fisica Generale "Amadeo Avogadro," Università degli Studi di Torino, I-10125 Torino, Italy

${ }^{17}$ Laboratoire Univers et Particules de Montpellier, Université Montpellier, CNRS/IN2P3, Montpellier, France

${ }^{18}$ Laboratoire Leprince-Ringuet, École polytechnique, CNRS/IN2P3, Palaiseau, France

${ }_{19}$ Consorzio Interuniversitario per la Fisica Spaziale (CIFS), I-10133 Torino, Italy

${ }^{20}$ INAF-Istituto di Astrofisica Spaziale e Fisica Cosmica, I-20133 Milano, Italy

${ }^{21}$ Agenzia Spaziale Italiana (ASI) Science Data Center, I-00133 Roma, Italy; sara.cutini@asdc.asi.it, stefano.ciprini@asdc.asi.it

${ }^{22}$ Istituto Nazionale di Fisica Nucleare, Sezione di Perugia, I-06123 Perugia, Italy

${ }^{23}$ Dipartimento di Fisica, Università degli Studi di Perugia, I-06123 Perugia, Italy

${ }^{24}$ College of Science, George Mason University, Fairfax, VA 22030, resident at Naval Research Laboratory, Washington, DC 20375, USA

25 INAF Osservatorio Astronomico di Roma, I-00040 Monte Porzio Catone (Roma), Italy

${ }^{26}$ Department of Physics, Stockholm University, AlbaNova, SE-106 91 Stockholm, Sweden

${ }^{27}$ The Oskar Klein Centre for Cosmoparticle Physics, AlbaNova, SE-106 91 Stockholm, Sweden; stefan@ astro.su.se

${ }^{28}$ The Royal Swedish Academy of Sciences, Box 50005, SE-104 05 Stockholm, Sweden

29 INAF Istituto di Radioastronomia, I-40129 Bologna, Italy

${ }^{30}$ Dipartimento di Astronomia, Università di Bologna, I-40127 Bologna, Italy

${ }^{31}$ Dipartimento di Fisica, Università di Udine and Istituto Nazionale di Fisica Nucleare, Sezione di Trieste, Gruppo Collegato di Udine, I-33100, Udine, Italy

${ }^{32}$ Università Telematica Pegaso, Piazza Trieste e Trento, 48, I-80132 Napoli, Italy

${ }^{33}$ Università di Udine, I-33100 Udine, Italy

${ }^{34}$ Dipartimento di Fisica "M. Merlin" dell’Università e del Politecnico di Bari, I-70126 Bari, Italy

Max-Planck-Institut für Radioastronomie, Auf dem Hügel 69, D-53121 Bonn, Germany

${ }^{36}$ Department of Physical Sciences, Hiroshima University, Higashi-Hiroshima, Hiroshima 739-8526, Japan

${ }^{37}$ Space Science Division, Naval Research Laboratory, Washington, DC 20375-5352, USA

${ }^{38}$ University of North Florida, Department of Physics, 1 UNF Drive, Jacksonville, FL 32224, USA

${ }^{39}$ School of Physics and Astronomy, University of Southampton, Highfield, Southampton, SO17 1BJ, UK

${ }^{40}$ Science Institute, University of Iceland, IS-107 Reykjavik, Iceland

${ }^{41}$ Department of Physics, Graduate School of Science, University of Tokyo, 7-3-1 Hongo, Bunkyo-ku, Tokyo 113-0033, Japan

${ }^{42}$ Department of Physics, KTH Royal Institute of Technology, AlbaNova, SE-106 91 Stockholm, Sweden

${ }^{43}$ Institute of Space Sciences (IEEC-CSIC), Campus UAB, E-08193 Barcelona, Spain 


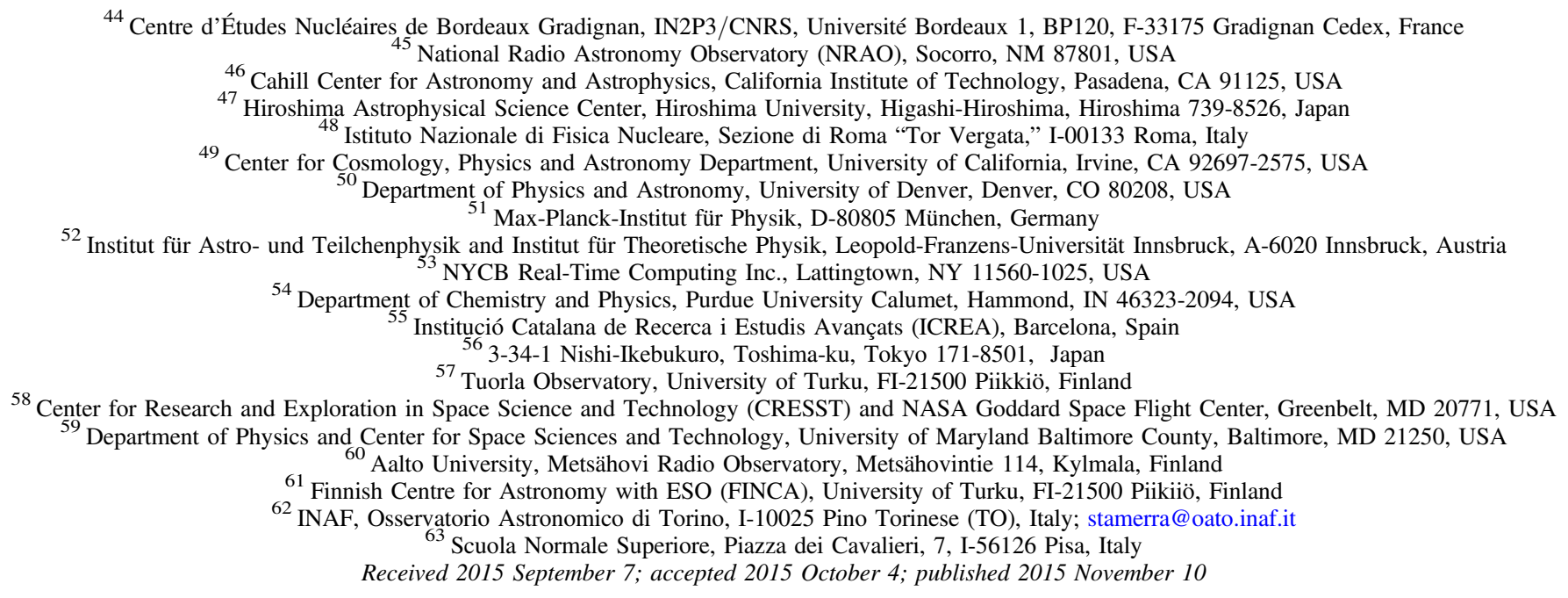

\section{ABSTRACT}

We report for the first time a $\gamma$-ray and multiwavelength nearly periodic oscillation in an active galactic nucleus. Using the Fermi Large Area Telescope we have discovered an apparent quasi-periodicity in the $\gamma$-ray flux $(E>100 \mathrm{MeV})$ from the $\mathrm{GeV} / \mathrm{TeV}$ BL Lac object PG $1553+113$. The marginal significance of the $2.18 \pm 0.08$ year period $\gamma$-ray cycle is strengthened by correlated oscillations observed in radio and optical fluxes, through data collected in the Owens Valley Radio Observatory, Tuorla, Katzman Automatic Imaging Telescope, and Catalina Sky Survey monitoring programs and Swift-UVOT. The optical cycle appearing in $\sim 10$ years of data has a similar period, while the $15 \mathrm{GHz}$ oscillation is less regular than seen in the other bands. Further long-term multiwavelength monitoring of this blazar may discriminate among the possible explanations for this quasi-periodicity.

Key words: accretion, accretion disks - BL Lacertae objects: general - BL Lacertae objects: individual

(PG 1553+113) - galaxies: jets - gamma rays: galaxies - gamma rays: general

\section{INTRODUCTION}

Among active galactic nuclei (AGNs), blazars are distinguished by erratic variability at all energies on a wide range of timescales. They are generally thought to be powered by supermassive black holes (SMBHs; $10^{8}-10^{9} M_{\odot}$ ). PG 1553 +113 (1ES 1553+113, $z \sim 0.49$; Danforth et al. 2010; Abramowski et al. 2015; Aliu et al. 2015) is an optically/Xray selected BL Lac object (Falomo \& Treves 1990) emitting variable $\mathrm{GeV} / \mathrm{TeV} \gamma$ radiation (Abramowski et al. 2015; Aleksic et al. 2015). As is typical in very high energy (VHE) BL Lacs, the energetic non-thermal emission of PG 1553+113 originates in a relativistic jet and has a spectral energy distribution (SED) with two humps, overwhelming any other component from either the nucleus or the host galaxy.

The Large Area Telescope (LAT) on the Fermi Gamma-ray Space Telescope is providing continuous monitoring of the high-energy $\gamma$-ray sky. The apparent modulation noted in the $\gamma$-ray flux of PG $1553+113$ initiated the multifrequency and long-term variability study described in this paper.

In Section 2 we describe the Fermi-LAT data analysis and the sources of multiwavelength data; Section 3 details the multiple approaches used for light curves and the crosscorrelation analysis; Section 4 outlines preliminary scenarios to interpret these results.

\footnotetext{
${ }^{64}$ NASA Postdoctoral Program Fellow, USA.

${ }^{65}$ Funded by contract FIRB-2012-RBFR12PM1F from the Italian Ministry of Education, University and Research (MIUR).
}

\section{FERMI-LAT AND RADIO, OPTICAL, AND X-RAY DATA}

The LAT is a pair conversion telescope with a $2.4 \mathrm{sr}$ field of view, sensitive to $\gamma$ rays from $\sim 20 \mathrm{MeV}$ to $>300 \mathrm{GeV}$ (Atwood et al. 2009). The present work uses the new Pass 8 LAT database (Atwood et al. 2013). The LAT operating mode allows it to cover the entire sky every two $\sim 1.6 \mathrm{hr}$ spacecraft orbits, providing a regular and uniform view of $\gamma$-ray sources and sampling timescales from hours to years. This work uses observations of PG $1553+113$ covering $\sim 6.9$ years $(2008$ August 4 to 2015 July 19; Modified Julian Day, MJD, 54682.65-57222.65). The LAT data analysis employed the standard ScienceTools v10r0p5 ${ }^{66}$ package, selecting events from $100 \mathrm{MeV}$ to $300 \mathrm{GeV}$ with P8R2_SOURCE_V6 instrument response functions, in a circular region of interest of $10^{\circ}$ radius centered on the position of PG $1553+113$. It used files gll_iem_v06 and iso_P8R2_SOURCE_V6_v06 to model the Galactic and isotropic diffuse emission. Contamination due to the $\gamma$-ray-bright Earth limb is avoided by excluding events with zenith angle $>90^{\circ}$. An unbinned maximum likelihood model fit technique is applied to each time bin with a power-law spectral model and photon index fixed to the 3FGL Catalog average value $(1.604 \pm 0.025$; Acero et al. 2015) for PG $1553+113$. The resulting light curves are shown in Figure 1.

Optical R-band data covering an interval of $\sim 9.9$ years (2005 April 19 to 2015 March 29; MJD 53479-57110) are reported in Figure 2. Most unpublished observations were

\footnotetext{
${ }^{66}$ http://fermi.gsfc.nasa.gov/ssc/data/analysis/documentation/
} 


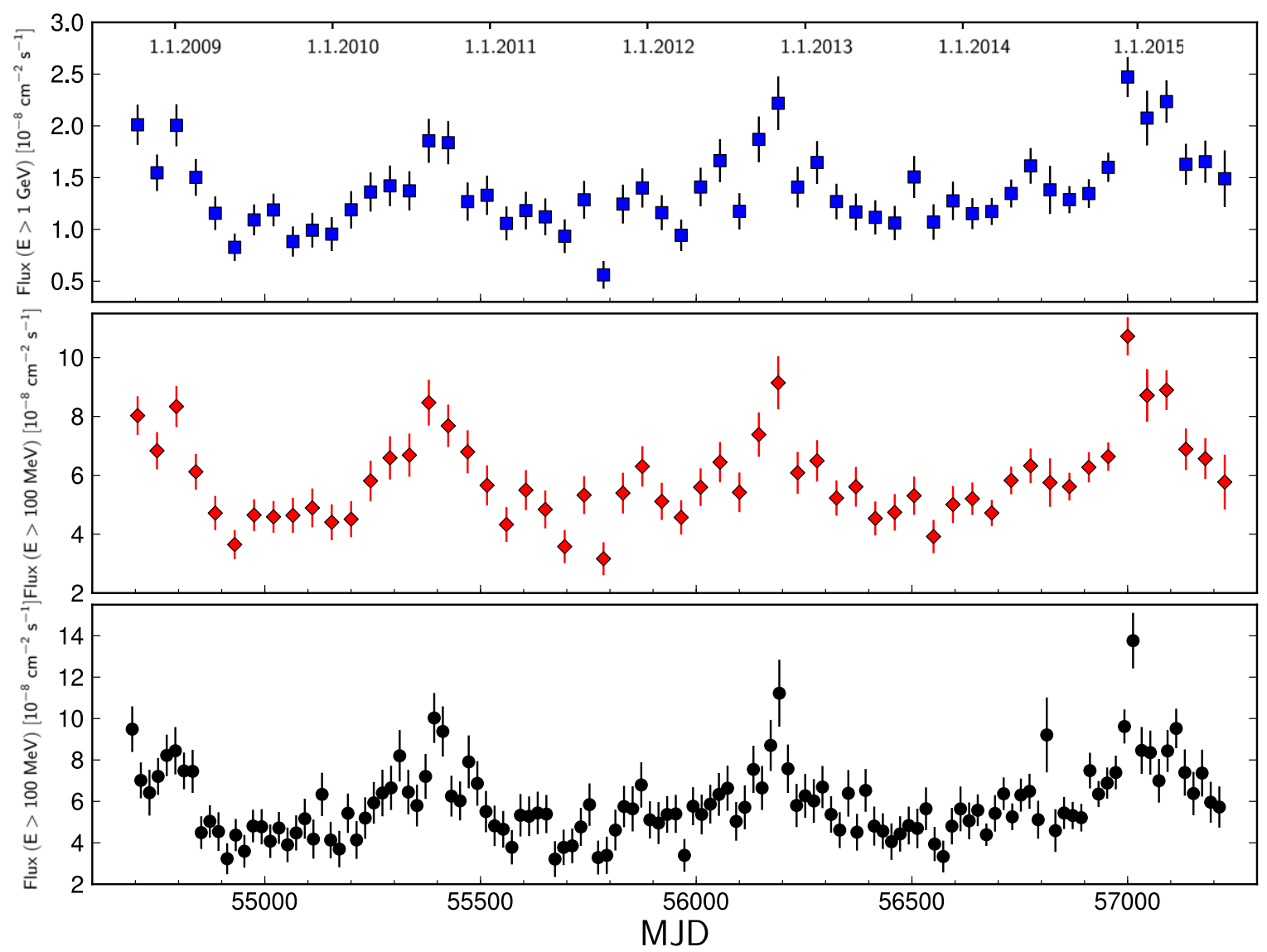

Figure 1. Fermi-LAT $\gamma$-ray light curves of PG $1553+113$ over $\sim 6.9$ years, from 2008 August 4 to 2015 July 19 . The light curve above 1 GeV is shown with a constant 45 day binning (top panel); two light curves above $100 \mathrm{MeV}$ are shown, with 45 and 20 day binning (middle and bottom panels).

performed as part of the Tuorla blazar monitoring program (Takalo et al. 2008). ${ }^{67}$ The data are reduced using a semiautomatic pipeline (K. Nilsson et al. 2015, in preparation). Public data from the Katzman Automatic Imaging Telescope (KAIT) and the Catalina Sky Survey (CSS) programs are also added. V-band magnitudes are scaled to the R-band values.

As part of an ongoing blazar monitoring program supporting Fermi (Richards et al. 2011), the Owens Valley Radio Observatory (OVRO) $40 \mathrm{~m}$ radio telescope has been observing PG $1553+113$ continually (about every 1-23 days) since 2008 August. Figure 2 reports published $15 \mathrm{GHz}$ light curves for the period from 2008 August 19 to 2014 May 18 (MJD 5469756795). OVRO instrumentation, data calibration, and reduction are described in Richards et al. (2011).

Swift observed PG 1553+113 110 times between 2005 April 20 and 2015 July 18 (unabsorbed $0.3-2 \mathrm{keV}$ flux light curve in Figure 2). X-Ray Telescope (XRT) data were first calibrated and cleaned (xrtpipeline, XRTDAS v.3.0.0) and then energy spectra were extracted from a region of 20 pixel $\left(\sim 47^{\prime \prime}\right)$ radius, with a nearby 20 pixel radius region as a background. Individual XRT spectra are well fitted with a log-parabolic model, with column density fixed to the Galactic value of $3.6 \times 10^{20} \mathrm{~cm}^{-2}$ (Kalberla et al. 2005). Aperture photometry ( 5 " radius) for the UVOT V-band filter was performed.

\footnotetext{
${ }^{67}$ http://users.utu.fi/kani/1m
}

\section{TEMPORAL VARIABILITY ANALYSIS AND CROSS-CORRELATION ANALYSIS}

We performed continuous wavelet transform (CWT) and Lomb-Scargle Periodogram (LSP) analyses on the light curves. Figure 3 shows clear peaks at $\sim 2$ years for $\gamma$-ray and optical power spectra. We also made an epoch folding (pulse shape) analysis to extract the period, shape, amplitude, and phase with uncertainties (Larsson 1996). The $\chi^{2}$ for the folded pulse as a function of the trial periods was fitted with a model containing four Fourier components, giving a period of $798 \pm 30$ days $(2.18 \pm 0.08$ years), consistent with the CWT and LSP findings (Figure 3). The value of the signal power peak does not change using regular 20 day and 45 day bins or an adaptive-bin technique (Lott et al. 2012) for construction of the LAT light curve.

A direct power density spectrum (PDS) constructed from a LAT count rate light curve using exposure-weighted aperture photometry (Corbet et al. 2007; Kerr 2011) above $100 \mathrm{MeV}$ for a region with $3^{\circ}$ radius with $600 \mathrm{~s}$ time bins (Figure 4 ) confirms previous results with a peak at $2.16 \pm 0.08$ years, at $82 \times$ the mean power level. The low-frequency modulation prevents an easy fit subtraction to the PDS continuum. The peak is $\sim 5$ times the mean level using a 4th order polynomial fit.

The significance of any apparent periodic variation depends on what assumption is made about spurious stochastic variability mimicking a periodic variation. The significance of the $\sim 2$ year $\gamma$-ray periodicity is difficult to assess given the limited length of the $\gamma$-ray light curve. Red noise, i.e., random and relatively enhanced low-frequency fluctuations over 


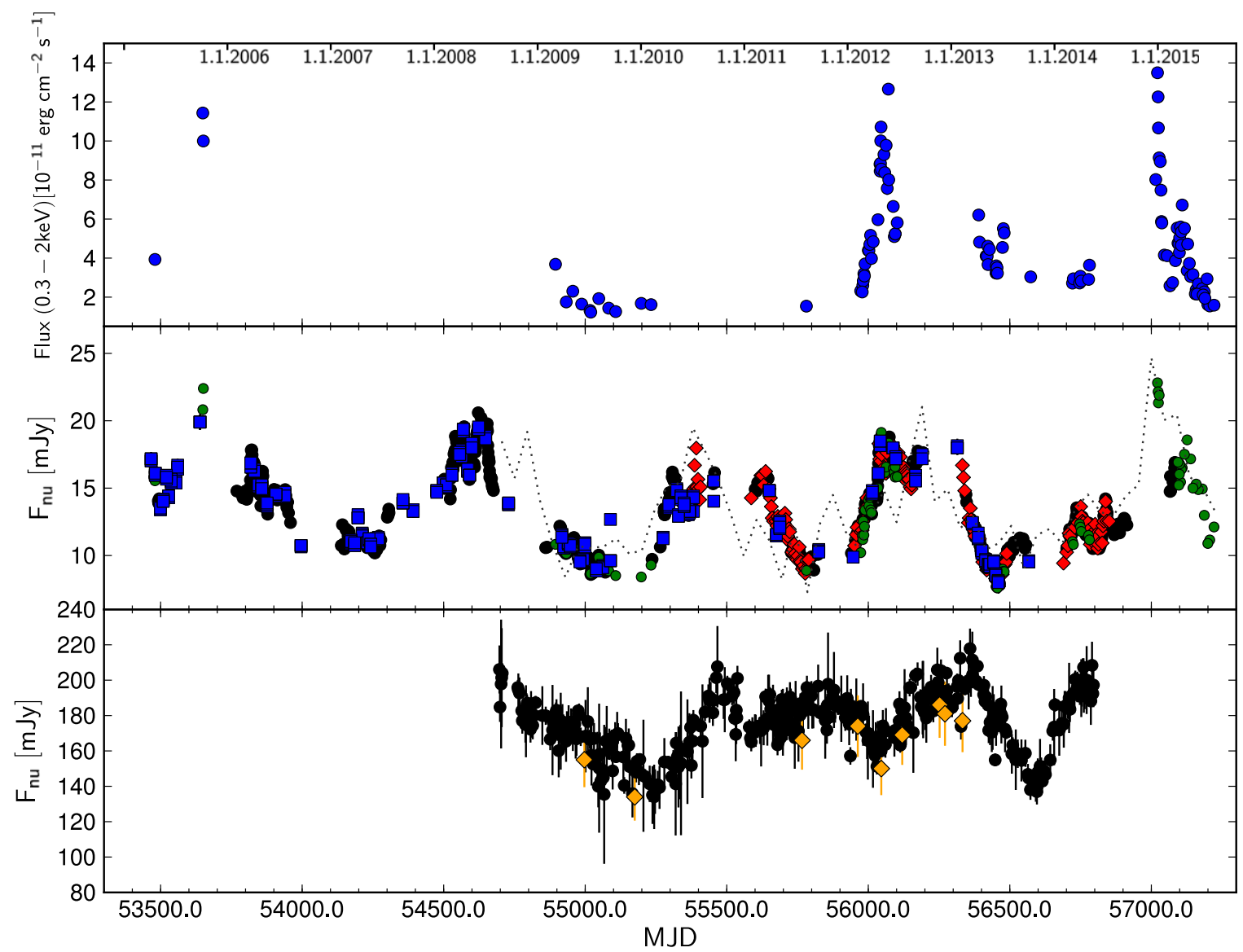

Figure 2. Multifrequency light curves of PG $1553+113$ at X-ray, optical, and radio bands. Top panel: Swift-XRT integrated flux (0.3-2.0 keV). Center panel: optical flux density from Tuorla (R filter, black filled circle points), Catalina CSS (V filter rescaled, blue filled squared points), KAIT (V filter rescaled, red filled diamond points), and Swift-UVOT (V filter rescaled, green filled circle points). Dotted line: LAT flux ( $E>100 \mathrm{MeV}$ ) with time bins of 20 days, scaled and $y$-shifted for comparison. Bottom panel: $15 \mathrm{GHz}$ flux density from OVRO $40 \mathrm{~m}$ (black filled circle points) and parsec-scale $15 \mathrm{GHz}$ flux density by VLBA (MOJAVE program, yellow diamond filled points).

intervals comparable to the sample length, hinders the evaluation of the periodicity significance (e.g., Hsieh et al. 2005; Lasky et al. 2015). We have approached the problem with two procedures.

(1) The red noise is assumed to be produced by similar amplitude flares (as seen in PG 1553+113 and some other LAT blazars), and the probability for these to line up in a regular pattern is estimated. The coherence of the periodic modulation was investigated by studying phase variations along the light curve. The local phase at each minimum and maximum was estimated by correlating a one-period-long data segment with the Fourier template of the full light curve. The rms variations relative to a perfectly coherent modulation were 27.4 days. The chance probabilities for three, four, and five random events to be distributed with at least this coherence, as estimated by Monte Carlo simulations, are 0.0535, 0.0105, and 0.0027, respectively, implying a chance probability of a few percent for the 3.5 peak $\gamma$-ray light curve of PG $1553+113$.

(2) We modeled the red noise using Monte Carlo simulations with a first-order autoregressive process as the null hypothesis to assess whether the signal is consistent with a stochastic origin. The nonlinear influence on the PDS is minimal thanks to the evenly spaced $\gamma$-ray light curve. The power peak in Figure 3 is above the $99 \%$ confidence contour level, i.e., has $<1 \%$ chance of being a statistical fluctuation. The optical power peak has $<5 \%$ chance of being a statistical fluctuation.
Although the $\gamma$-ray periodicity signal alone is not compelling, the 9.9 years of optical data support the finding of a periodic oscillation in PG $1553+113$. The optical data, although affected by seasonal gaps, were analyzed using the same techniques as for the $\gamma$-ray data. This analysis gives a period of $754 \pm 20$ days ( $2.06 \pm 0.05$ years), consistent within uncertainties with the $\gamma$-ray results (Figure 3).

The less coherent $15 \mathrm{GHz}$ light curve (5.7 years OVRO data) shows a signal power peak at $1.9 \pm 0.1$ year, with an additional power component at a 1.2 year timescale. Swift-XRT data show a factor of 5 variation linearly correlated with the $\gamma$-ray flux, while the synchrotron peak frequency shows a factor of $\sim 6$ increase during high X-ray states, as suggested by Reimer et al. (2008).

The long-term X-ray count rate light curve from the $R X T E$ ASM instrument (1996 February 20 to 2010 September 11) and the Swift-BAT (from 2005 May 29) were also analyzed but do not show any signal above the low-frequency noise because of insufficient statistics.

An important diagnostic for multifrequency periodicity analysis is the discrete cross-correlation function (DCCF) used with two independent and complementary approaches.

In the first procedure, flux variations are modeled assuming a simple power law $\propto 1 / f^{\alpha}$ (with $f=1 / t$ ) in the PDS as measured directly from the light curve data, allowing us to estimate the cross-correlation significance, and avoiding the 

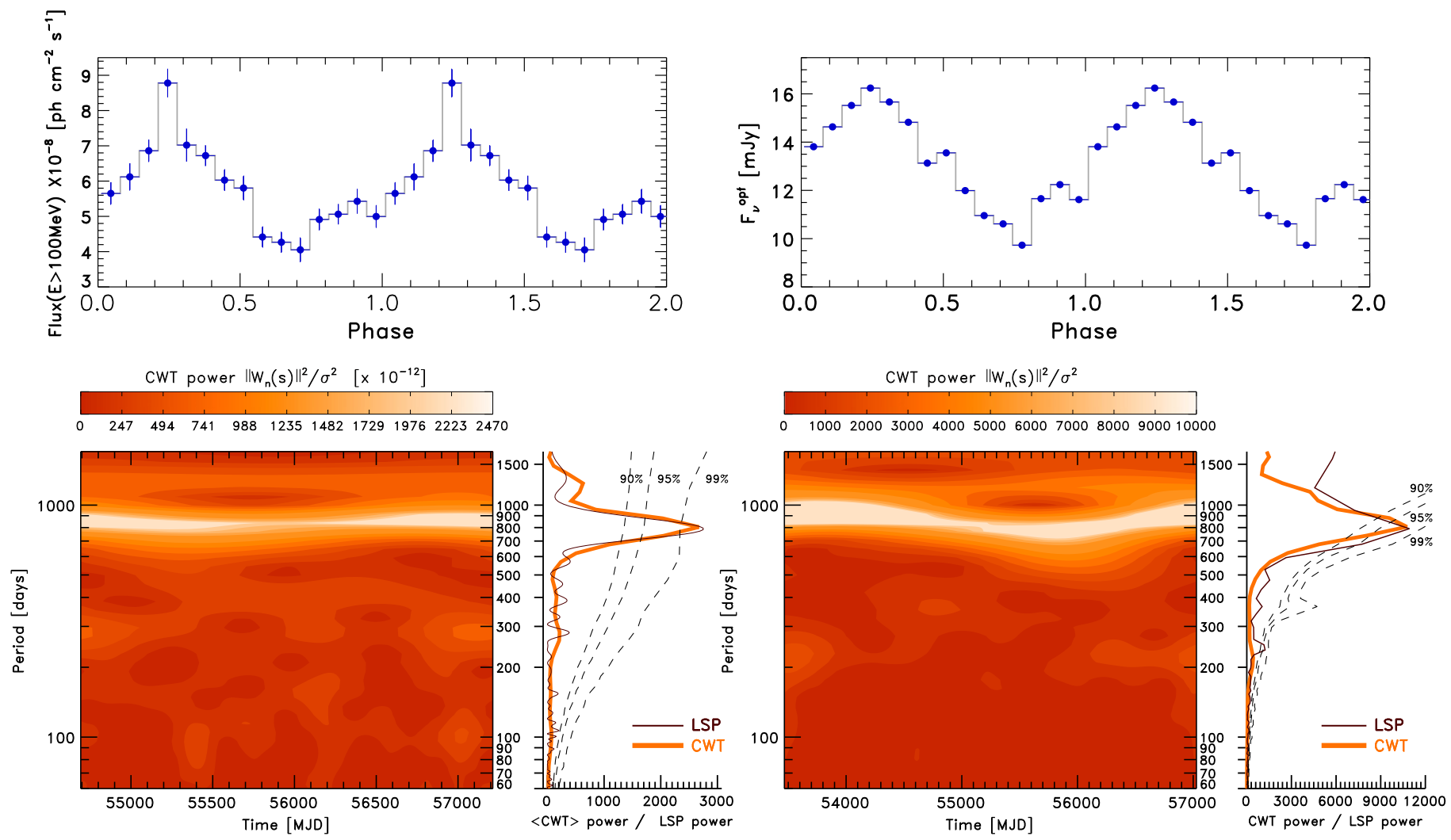

Figure 3. Left top panel: pulse shape (epoch-folded) $\gamma$-ray $(E>100 \mathrm{MeV})$ flux light curve at the 2.18 year period (two cycles shown). Left bottom panels: $2 \mathrm{D}$ plane contour plot of the CWT power spectrum (scalogram) of the $\gamma$-ray light curve using a Morlet mother function (filled color contour). The side panel to this is the 1D smoothed, all-epoch averaged, spectrum of the CWT scalogram showing a signal power peak in agreement with the 2.18 year value, also showing the LSP. Dashed lines depict increasing levels of confidence against red noise calculated with Monte Carlo simulations. The $\gamma$-ray signal peak is above the $99 \%$ confidence contour level ( $<1 \%$ chance probability of being spurious). Right top panel: pulse shape from epoch folding of the optical flux light curve at the 2.18 year period (two cycles shown). Right bottom panels: the same CWT and LSP diagrams for the optical light curve. The optical signal peak is above the $95 \%$ confidence contour level.

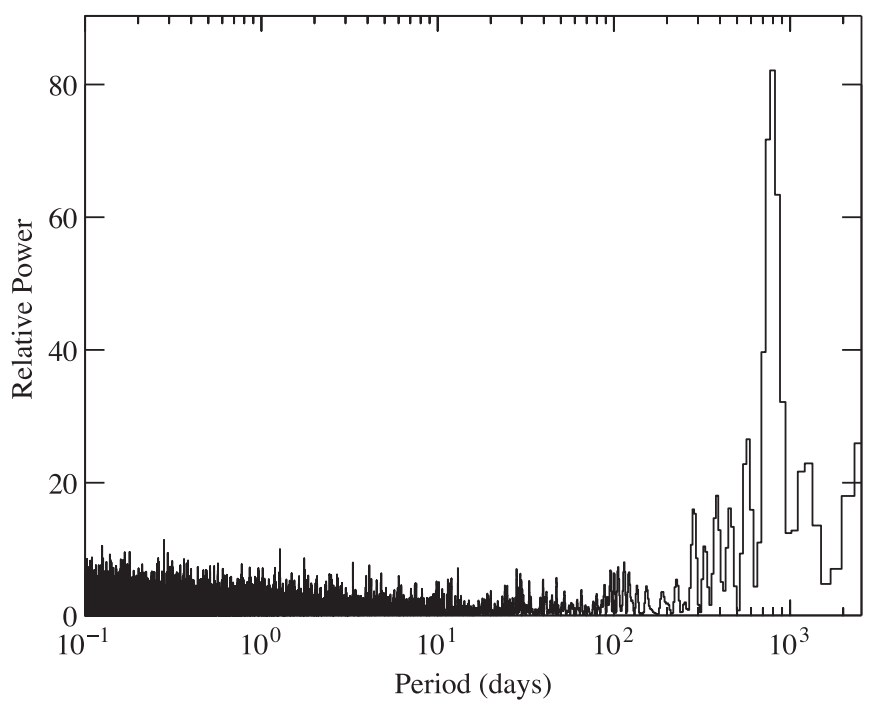

Figure 4. Power density spectrum (PDS) of the LAT $0.1-300 \mathrm{GeV}$ count rate light curve of PG $1553+113$ from a $3^{\circ}$ exposure-weighted aperture photometry technique with $600 \mathrm{~s}$ time bins.

assumption of equal variability in all sources at the cost of a model assumption (Max-Moerbeck et al. 2014). For the $\gamma$-ray light curve with 20 day binning we obtain a best fit $\alpha=0.8$, but the error is unconstrained, indicating that the length of the data set is too short (i.e., below five cycles) relative to the suspected periodic modulation to enable a reliable data characterization.
The 45 day bin light curve yields a best fit $\alpha=0.1$ with unconstrained error. The optical PSD is constrained: the best fit value is $\alpha=1.85$, with $1 \sigma$ limits at $[1.75,2.00]$. The $15 \mathrm{GHz}$ flux light curve has a slope of $\alpha=1.4$, with unconstrained limits on the $\alpha$ values as for the $\gamma$-ray data. The DCCF between the unbinned radio light curve and the 20 day bin $\gamma$-ray light curve results in a most probable time lag for the radio flux lagging the $\gamma$-ray flux by $50 \pm 20$ days, with $98.14 \%$ significance for the best PSD fit with a range of $[89.56 \%-$ $99.99 \%$ ] when fit errors are taken into account (Figure 5) using the fitting procedure of Max-Moerbeck et al. (2014). The DCCF between the unbinned optical light curve and the 20 day bin $\gamma$-ray light curve results in a most probable time lag for the $\gamma$-ray flux lagging the optical flux by $130 \pm 14$ days, with 99.14\% significance for the best PSD fit and [96.09\%-99.97\%] when fit errors are taken into account (Figure 5). The DCCF peak is broad, however, and consistent with no lag. This is also seen when the optical data are rebinned into 20 day intervals, as shown in the bottom panel, where the most probable lag is $10 \pm 51$ days.

In the second procedure, the significance of the $\gamma$-ray-radio correlation was estimated to be $95 \%$ using a mixed source correlation procedure (Fuhrmann et al. 2014), cross-correlating the PG 1553+113 light curve with those of 132 comparison sources in that work, and evaluating the average DCCF level for time lags of -100 to +100 days. The $\gamma$-ray-optical correlation is significant at the $99 \%$ level, even though it is partly limited by the number of comparison sources and optical 

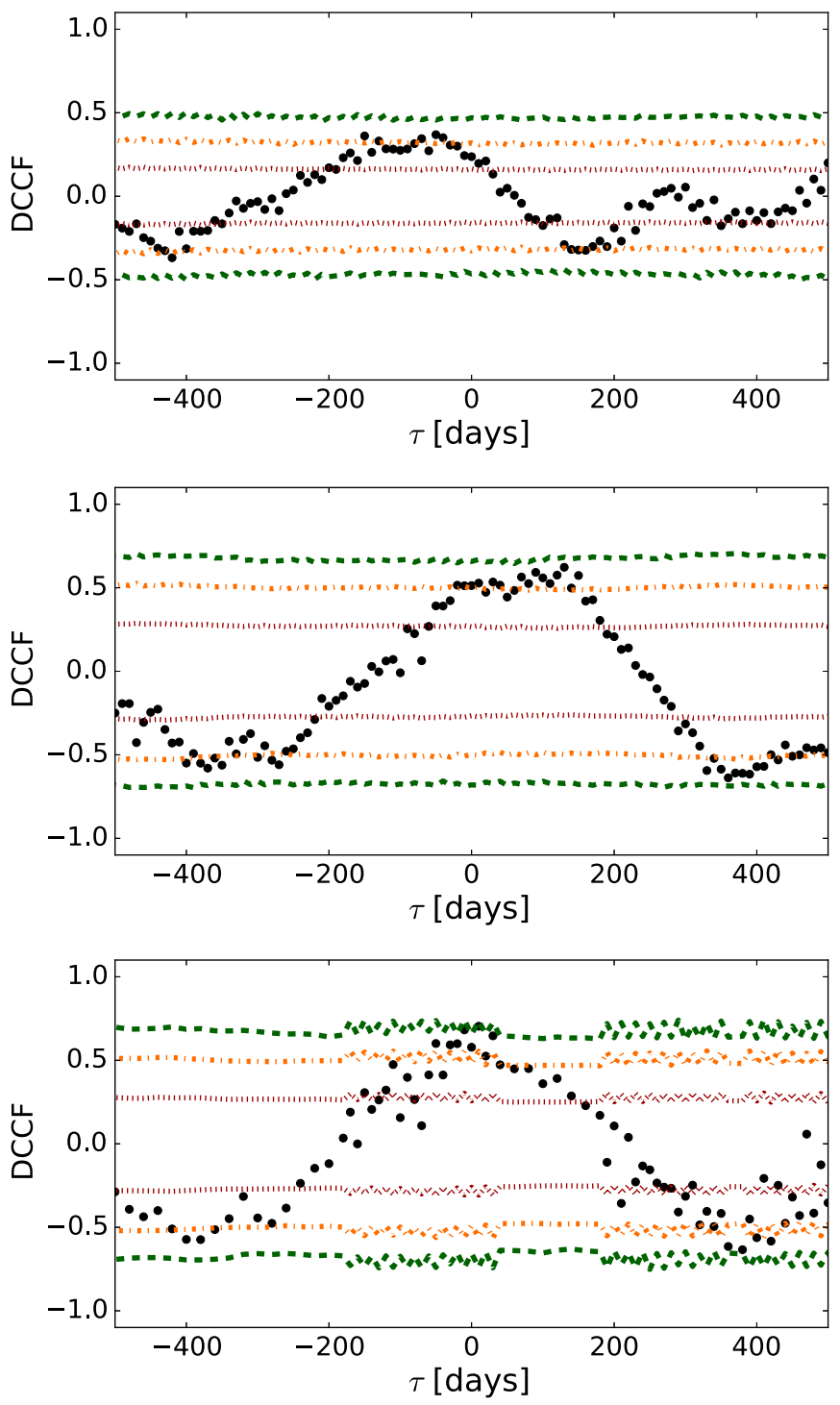

Figure 5. Discrete cross-correlation plots from the approach with the PDS model measured from the light curve data (Max-Moerbeck et al. 2014). In each plot the black dots are the DCCF estimates and the red, orange, and green lines are the $1 \sigma, 2 \sigma$, and $3 \sigma$ significance levels, respectively. Top panel: DCCF between the radio $15 \mathrm{GHz}$ and $\gamma$-ray (20 day time bins) light curve. Central panel: DCCF between the unbinned optical light curve and $\gamma$-ray (20 day time bins) light curve. Bottom panel: DCCF between the 20 day rebinned optical light curve and $\gamma$-ray (20 day time bins) light curve. The oscillating shape of the significance contours in this case is due to the number of samples in each bin.

light curve gaps. With only 132 comparison light curves we can measure a minimum probability value of 0.0075 , therefore in principle a $99 \%$ level of significance, but in this approach the error in that estimate is hard to determine. With the mixed source methods there are two limitations: (1) the assumption that all sources can be described with the same model for the variability, and (2) the sample variance due to the limited number of light curves must be assessed. The optical flux is found to lead the $\gamma$-ray variations by $75 \pm 27$ days and the radio by $158 \pm 10$ days ( $\gamma$-ray variations lead the $15 \mathrm{GHz}$ flux variations by $83 \pm 27$ days). The possible reverse $\gamma$-ray-optical time lag decreases to $28 \pm 27$ days when the optical light curve is binned.

The possible optical- $\gamma$-ray lag was already pointed out by Cohen et al. (2014) using KAIT unbinned optical light curves and LAT data. The high degree of $\gamma$-ray-radio correlation in PG $1553+113$ is not typically found in other individual blazars/AGNs (see Max-Moerbeck et al. 2014). Significant cross-correlations are, nevertheless, found when stacking blazar samples (radio lagging $\gamma$-rays; Fuhrmann et al. 2014).

\section{DISCUSSION AND CONCLUSIONS}

Factors that led to the indication of a possible $\sim 2$ year periodic modulation in PG $1553+113$ are the continuous allsky survey of Fermi, the increased capability of the new FermiLAT Pass 8 data, and the long-term radio/optical monitoring of $\gamma$-ray blazars. Although the statistical significance of periodicity is marginal in each band, the consistent positive crosscorrelation between bands strengthens the case, making PG $1553+113$ the first possible quasi-periodic $\mathrm{GeV} \gamma$-ray blazar and a prime candidate for further studies. Hints of possible $\gamma$-ray periodicities are rare in the literature (for example Sandrinelli et al. 2014). The similarity of the low- and highenergy modulation in PG $1553+113$ is also a novel behavior for AGNs (Rieger 2004, 2007). Any periodic driving scenario should be related to the relativistic jet itself or to the process feeding the jet for this VHE BL Lac object. We outline, as examples, four possibilities.

1. Pulsational accretion flow instabilities, approximating periodic behavior, are able to explain modulations in the energy outflow efficiency. Magnetically arrested and magnetically dominated accretion flows (MDAFs) could be suitable regimes for radiatively inefficient BL Lacs (Fragile \& Meier 2009), characterized by advectiondominated accretion flows and subluminal, turbulent, and peculiar radio kinematics (Kharb et al. 2008; Karouzos et al. 2012; Piner \& Edwards 2014). Such kinematics are sometimes explained as a precessing or helical jet (Conway \& Murphy 1993). MDAFs in an inner disk portion are able to efficiently impart energy to particles in the jets of VHE BL Lacs (Tchekhovskoy et al. 2011). Periodic instabilities are believed to have short periods, $\sim 10^{5} \mathrm{~s} \cdot\left(M_{\mathrm{SMBH}} / 10^{8} M_{\odot}\right)$ (Honma et al. 1992), but MHD simulations of magnetically choked accretion flows are seen to produce longer periods for slow-spinning SMBHs (McKinney et al. 2012).

2. Jet precession (e.g., Romero et al. 2000; Stirling et al. 2003; Caproni et al. 2013), rotation (Camenzind \& Krockenberger 1992; Vlahakis \& Tsinganos 1998; Hardee \& Rosen 1999), or helical structure (e.g., Conway \& Murphy 1993; Roland et al. 1994; Villata \& Raiteri 1999; Nakamura \& Meier 2004; Ostorero et al. 2004; Mohan \& Mangalam 2015), i.e., geometrical models (Rieger 2004), in the presence of a jet wrapped by a sufficiently strong magnetic field, could have a net apparent periodicity from the change of the viewing angle. Correspondingly, the resulting Doppler magnification factor changes periodically without the need for intrinsic variation in outflows and efficiency. Non-ballistic hydrodynamical jet precession may explain variations with periods $>1$ year (Rieger 2004). A differential Doppler factor $\Delta \mathcal{D}(t)=$ $\Gamma^{-1}(1-\beta(t) \cos \theta(t))^{-1} \lesssim 40 \%$ variation (precession angle $\sim 1^{\circ}$ ) might be sufficient to support the $\sim 2.8$ amplitude flux modulation seen in $\gamma$ rays. A homogeneous curved helical jet scenario for PG $1553+113$ was proposed in Raiteri et al. (2015). 
3. A mechanism analogous to low-frequency QPO from Galactic high-mass binaries/microquasars could produce an accretion-outflow coupling mechanism as the basis of the periodicity (Fender \& Belloni 2004). King et al. (2013) ascribed the radio QPO in the FSRQ CGRaBS $\mathrm{J} 1359+4011$ to this mechanism. However BL Lac objects like PG $1553+113$ are thought to possess a lower accretion rate. The microquasar QPO mechanism of Lense-Thirring precession (Wilkins 1972) requires that the inner accretion flow forms a geometrically thick torus rather than a standard thin disk as the latter warps (Bardeen-Petterson effect, Bardeen \& Petterson 1975) rather than precesses (Ingram et al. 2009). A low mass accretion rate means that the accretion process probably forms an advection-dominated accretion flow (ADAF) so it can precess (Fragile \& Meier 2009). The X-ray emission in PG $1553+113$ is probably from the jet rather than from the flow, making it unlikely that the changing inclination of the hot flow causes the QPO. However, Lense-Thirring precession of the flow could affect the jet direction, giving the QPO as in (2) above.

4. The presence of a gravitationally bound binary SMBH system (Begelman et al. 1980; Barnes \& Hernquist 1992) with a total mass of $\sim 10^{8} M_{\odot}$ and a milliparsec separation in the early inspiral gravitational-wave driven regime might be another hypothesis. Keplerian binary orbital motion would induce periodic accretion perturbations (Valtonen et al. 2008; Pihajoki et al. 2013; Liu et al. 2015) or jet nutation expected from the misalignment of the rotating SMBH spins or the gravitational torque on the disk exerted by the companion (Katz 1997; Romero et al. 2000; Caproni et al. 2013; Graham et al. 2015). Significant acceleration of the disk evolution and accretion onto a binary SMBH system is depicted by modeling (Nixon et al. 2013; Doğan et al. 2015).

Binary SMBH induced periodicities have timescales ranging from $\sim 1$ to $\sim 25$ years (Komossa 2006; Rieger 2007). The SMBH total mass in PG $1553+113$, estimated utilizing the putative link between inflow/ accretion (disk luminosity) and outflow/jet (jet power) in blazars (Ghisellini et al. 2014), is $\simeq 1.6 \times 10^{8} M_{\odot}$ using a $0.1 \dot{M}_{\text {Edd }}$ rate and Doppler factor $\mathcal{D}=30$, in agreement with estimates for VHE BL Lacs (Woo et al. 2005).

The observed 2.18 year period is equivalent to an intrinsic orbital time $T_{\mathrm{Kep}}^{\prime} \leqslant T_{\mathrm{obs}} /(1+z) \simeq 1.5$ years, and the binary system size would be $0.005 \mathrm{pc}(\sim 100$ Schwarzschild radii). The probability of observing such a milliparsec system, estimated from the binary mass ratios $\sim 0.1-0.01$ and the GW-driven regime lifetime (Peters 1964), $t_{\mathrm{GW}} \simeq 10^{5}-10^{6}$ years, might be too small.

Periodicities claimed for AGNs are often controversial; however, PG $1553+113$ may potentially represent a key $\gamma$-ray/ multimessenger laboratory in the hypothesis of low-frequency gravitational-wave emission and may have associated $\mathrm{PeV}$ neutrino emission (Padovani \& Resconi 2014). Very long baseline interferometry structure observations (Kharb et al. 2008), radio/optical polarization data, and a prolonged multifrequency monitoring campaign will shed light on the situation. If the periodic modulation is real and coherent, as would be expected for a binary scenario, then subsequent maxima would be expected in 2017 and 2019, well within the possible lifetime of the Fermi mission.
We thank the anonymous referee for useful and constructive comments. We extend special thanks to Prof. C. Done of Durham University, UK, and Prof. R. W. Romani of Stanford University, USA, for useful comments during the course of this work. The Fermi-LAT Collaboration acknowledges support for LAT development, operation and data analysis from NASA and DOE (United States); CEA/Irfu and IN2P3/CNRS (France); ASI and INFN (Italy); MEXT, KEK, and JAXA (Japan); and the K.A. Wallenberg Foundation, the Swedish Research Council, and the National Space Board (Sweden). Science analysis support in the operations phase from INAF (Italy) and CNES (France) is also gratefully acknowledged. The Tuorla blazar monitoring program has been partially supported by the Academy of Finland grant 127740 . The KAIT telescope program is supported by Katzman Foundation and the National Science Foundation. The CSS survey is funded by the National Aeronautics and Space Administration under grant No. NNG05GF22G issued through the Science Mission Directorate Near-Earth Objects Observations Program. The CRTS survey is supported by the U.S. National Science Foundation under grants AST-0909182. The OVRO $40 \mathrm{~m}$ program is supported in part by NASA grants NNX08AW31G and NNX11A043G and NSF grants AST-0808050 and AST1109911. The MOJAVE program is supported under NASAFermi grant NNX12A087G. The National Radio Astronomy Observatory (NRAO) is a facility of the National Science Foundation operated under cooperative agreement by Associated Universities, Inc. The NASA Swift $\gamma$-ray burst explorer is a MIDEX Gamma Ray Burst mission led by NASA with participation of Italy and the UK. This research has made use of the Smithsonian/NASA's ADS bibliographic database. This research has made use of the NASA/IPAC NED database (JPL CalTech and NASA, USA). This research has made use of the archives and services of the ASI Science Data Center (ASDC), a facility of the Italian Space Agency (ASI Headquarters, Rome, Italy). This research has made use of the XRT Data Analysis Software (XRTDAS) developed under the responsibility of the ASDC. This work is a product of the ASDC Fermi team developed in the frame of the INAF Senior Scientists project and the foreign visiting scientists program of ASDC.

Facilities: Fermi Gamma-ray Space Telescope, Swift, OVRO:40m, KAIT.

\section{REFERENCES}

Abramowski, A., Aharonian, F., Ait Benkhali, F., et al. 2015, ApJ, 802, 65 Acero, F., Ackermann, M., Ajello, M., et al. 2015, ApJS, 218, 23

Aleksić, J., Ansoldi, S., Antonelli, L. A., et al. 2015, MNRAS, 450, 4399 Aliu, E., Archambault, S., Archer, A., et al. 2015, ApJ, 800, 61

Atwood, W., Albert, A., Baldini, L., et al. 2013, in 2012 Fermi Symposium Proc., eConf. C121028, 8 (arXiv:1303.3514)

Atwood, W. B., Abdo, A. A., Ackermann, M., et al. 2009, ApJ, 697, 1071 Bardeen, J. M., \& Petterson, J. A. 1975, ApJL, 195, L65

Barnes, J. E., \& Hernquist, L. 1992, ARA\&A, 30, 705

Begelman, M. C., Blandford, R. D., \& Rees, M. J. 1980, Natur, 287, 307 Camenzind, M., \& Krockenberger, M. 1992, A\&A, 255, 59

Caproni, A., Abraham, Z., \& Monteiro, H. 2013, MNRAS, 428, 280

Cohen, D. P., Romani, R. W., Filippenko, A. V., et al. 2014, ApJ, 797, 137

Conway, J. E., \& Murphy, D. W. 1993, ApJ, 411, 89

Corbet, R. H. D., Markwardt, C. B., \& Tueller, J. 2007, ApJ, 655, 458

Danforth, C. W., Keeney, B. A., Stocke, J. T., Shull, J. M., \& Yao, Y. 2010, ApJ, 720, 976

Doğan, S., Nixon, C., King, A., \& Price, D. J. 2015, MNRAS, 449, 1251

Falomo, R., \& Treves, A. 1990, PASP, 102, 1120

Fender, R., \& Belloni, T. 2004, ARA\&A, 42, 317

Fragile, P. C., \& Meier, D. L. 2009, ApJ, 693, 771

Fuhrmann, L., Larsson, S., Chiang, J., et al. 2014, MNRAS, 441, 1899 
Ghisellini, G., Tavecchio, F., Maraschi, L., Celotti, A., \& Sbarrato, T. 2014, Natur, 515, 376

Graham, M. J., Djorgovski, S. G., Stern, D., et al. 2015, Natur, 518, 74

Hardee, P. E., \& Rosen, A. 1999, ApJ, 524, 650

Honma, F., Matsumoto, R., \& Kato, S. 1992, PASJ, 44, 529

Hsieh, C.-H., Glaser, S. M., Lucas, A. J., \& Sugihara, G. 2005, Natur, 435, 336

Ingram, A., Done, C., \& Fragile, P. C. 2009, MNRAS, 397, L101

Kalberla, P. M. W., Burton, W. B., Hartmann, D., et al. 2005, A\&A, 440, 775

Karouzos, M., Britzen, S., Witzel, A., Zensus, J. A., \& Eckart, A. 2012, A\&A, 537, AA112

Katz, J. I. 1997, ApJ, 478, 527

Kerr, M. 2011, ApJ, 732, 38

Kharb, P., Gabuzda, D., \& Shastri, P. 2008, MNRAS, 384, 230

King, O. G., Hovatta, T., Max-Moerbeck, W., et al. 2013, MNRAS, 436, L114

Komossa, S. 2006, MmSAI, 77, 733

Larsson, S. 1996, A\&AS, 117, 197

Lasky, P. D., Melatos, A., Ravi, V., \& Hobbs, G. 2015, MNRAS, 449, 3293

Liu, T., Gezari, S., Heinis, S., et al. 2015, ApJL, 803, L16

Lott, B., Escande, L., Larsson, S., \& Ballet, J. 2012, A\&A, 544, A6

Max-Moerbeck, W., Hovatta, T., Richards, J. L., et al. 2014, MNRAS, 445,428

McKinney, J. C., Tchekhovskoy, A., \& Blandford, R. D. 2012, MNRAS, 423, 3083

Mohan, P., \& Mangalam, A. 2015, ApJ, 805, 91

Nakamura, M., \& Meier, D. L. 2004, ApJ, 617, 123

Nixon, C., King, A., \& Price, D. 2013, MNRAS, 434, 1946
Ostorero, L., Villata, M., \& Raiteri, C. M. 2004, A\&A, 419, 913

Padovani, P., \& Resconi, E. 2014, MNRAS, 443, 474

Peters, P. C. 1964, PhRv, 136, 1224

Pihajoki, P., Valtonen, M., \& Ciprini, S. 2013, MNRAS, 434, 3122

Piner, B. G., \& Edwards, P. G. 2014, ApJ, 797, 25

Raiteri, C. M., Stamerra, A., Villata, M., et al. 2015, MNRAS, 454, 353

Reimer, A., Costamante, L., Madejski, G., Reimer, O., \& Dorner, D. 2008, ApJ, 682, 775

Richards, J. L., Max-Moerbeck, W., Pavlidou, V., et al. 2011, ApJS, 194, 29

Rieger, F. M. 2004, ApJL, 615, L5

Rieger, F. M. 2007, Ap\&SS, 309, 271

Roland, J., Teyssier, R., \& Roos, N. 1994, A\&A, 290, 357

Romero, G. E., Chajet, L., Abraham, Z., \& Fan, J. H. 2000, A\&A, 360, 57

Sandrinelli, A., Covino, S., \& Treves, A. 2014, ApJL, 793, LL1

Stirling, A. M., Cawthorne, T. V., Stevens, J. A., et al. 2003, MNRAS, 341, 405

Takalo, L. O., Nilsson, K., Lindfors, E., et al. 2008, in AIP Conf. Ser. 1085 , Proceedings of the 4th International Meeting on High Energy Gamma-Ray Astronomy (Melville, NY: AIP), 705

Tchekhovskoy, A., Narayan, R., \& McKinney, J. C. 2011, MNRAS, 418, L79

Valtonen, M. J., Lehto, H. J., Nilsson, K., et al. 2008, Natur, 452, 851

Villata, M., \& Raiteri, C. M. 1999, A\&A, 347, 30

Vlahakis, N., \& Tsinganos, K. 1998, MNRAS, 298, 777

Wilkins, D. C. $1972, \mathrm{PhRvD}, 5,814$

Woo, J.-H., Urry, C. M., van der Marel, R. P., Lira, P., \& Maza, J. 2005, ApJ, 631,762 